\title{
CRESCIMENTO DIFERENCIAL DE LINHAGENS DE MILHO EM SOLUÇÃO NUTRITIVA COM BAIXO NIVEL DE POTÁSSIO (')
}

\author{
ANGELA MARIA CANGIANI FURLANI $\left({ }^{2}\right)$, ONDINO CLEANTE BATAGLIA $\left({ }^{3}\right)$ \\ e MARLENE LIMA $\left({ }^{4}\right)$
}

\section{RESUMO}

Dois ensaios foram conduzidos no Centro Experimental de Campinas, no período agosto-outubro de 1983, em condiçōes de casa de vegetação, para avaliar e selecionar linhagens de milho (Zea mays L.) quanto à eficiência na absorção e utilizaçāo de potássio em solução nutritiva. No primeiro ensaio, seis linhagens foram cultivadas com $20,40,60,80$ e $100 \mathrm{mg} /$ litro de $\mathrm{K}$ até aos 34 dias de idade, com o objetivo de determinar o nível adequado para diferenciação das plantas. No segundo, 37 linhagens de milho foram selecionadas com $20 \mathrm{mg} /$ litro de $\mathrm{K}$ ate aos 25 dias de idade. As soluçōes nutritivas foram continuamente arejadas e não renovadas e as plantas, deixadas crescer até aparecerem sintomas de deficiência de potássio nas folhas inferiores. As variaçōes observadas nos pesos de matéria seca das raízes (CV das médias $=38,9 \%)$ foram maiores que aquelas da parte aérea (CV das médias = $28,5 \%$ ). As linhagens foram classificadas de acordo com a produção de matéria seca total, em grupos eficientes, ineficientes e medianamente eficientes, utilizando-se de um intervalo de confiança para a média geral. A absorção de

(1) Trabalho financiado pelo Convênio FINEP/FNDCT/SAA. Apresentado na XVII Reuniāo Brasileira de Fertilidade do Solo, realizado em Londrina, PR, no perfodo de $27 / 7$ a 1\%/8/86. Recebido para publicaçāo em 20 de fevereiro de 1986.

(2) Pesquisadora Cientifica da Seçāo de Fertilidade do Solo e Nutrição de Plantas, Instituto Agronômico (IAC), Caixa Postal 28, 13001 Campinas (SP). Com bolsa de suplementação do CNPq. CNPq.

(3) Pesquisador Científico da Seção de Fertilidade do Solo, IAC. Com bolsa de suplementação do

(4) Pesquisadora Científica da Seção de Genética, IAC. 
$\mathrm{K}$ pelas linhagens, avaliada pelo seu conteúdo total, variou (CV das médias = 9\%) acompanhando a variação observada nos pesos de matéria seca total ( $\mathrm{r}=$ 0,92). Entretanto, a relação de eficiência das linhagens apresentou variação maior (CV das médias $=23 \%$ ) e também acompanhou a variaçáo no crescimento das plantas $(r=0,99)$. Isso é uma indicação de que o mecanismo de utilizaçāo de $\mathrm{K}$ pelas plantas foi o fator que mais contribuiu para a diferenciação entre os genótipos.

Termos de indexação: potássio, absorção, conteúdo, eficiência; milho, linhagens, solução nutritiva.

\section{INTRODUÇÃO}

As características morfológicas e fisiológicas das raizes de milho e de outras plantas influem decisivamente na eficiência de absorção e utilização de nutrientes como o fósforo e o potássio; que dependem principalmente do mecanismo de difusão para serem transportados até as raizes.

Muitos pesquisadores afirmam que plantas mais eficientes na absorção de $\mathrm{P}$ e $\mathrm{K}$, possuem sistemas radiculares mais desenvolvidos, com raizes mais finas e ramificadas e com parâmetros fisiológicos de absorção ( $\mathrm{Km}$, Vmax) diferentes (SCHENK \& BARBER, 1980; BALIGAR, 1985; OLIVEIRA \& MIELNICZUK, 1978; BALIGAR \& BARBER, 1979; GLASS \& PERLEY, 1980; GLASS et alii, 1981; FURLANI et alii, 1983; FURLANI et alii 1984a, b; FURLANI et alii, 1985a).

Entretanto, dependendo da espécie e do nutriente em estudo, as características morfológicas das raizes e a eficiência no processo de utilização do nutriente são mais importantes na diferenciação de cultivares do que os parâmetros fisiológicos da absorção em si (SHEA et alii, 1968; MAKMUR et alii, 1978; ANNUAL REPORT, 1981; CLARK, 1983; FURLANI et alii, 1985b; FURLANI et alii, 1986).

Todos esses mecanismos que conferem maior eficiência à planta são reconhecidamente herdáveis. Assim, em milho, linhagens e híbridos que apresentavam maiores potenciais de aumento na velocidade de absorção de $\mathrm{K}$ quando submetidos a um estresse em solução nutritiva, apresentavam também maiores* produçōes de grãos quando cultivados em campo, em baixo nível de K. Esta caracteristica mostrou-se geneticamente controlada e só se expressava em condiçōes de estresse (FRICK \& BAUMAN, 1978, 1979). Diferenças nos parâmetros de absorção de $\mathrm{P}$ e $\mathrm{K}$ (Km e V́max) entre genótipos de milho, mostraram estar sob controle genético (BALIGAR \& BARBER, 1979). Diferenças no mecanismo de utilização de $\mathrm{K}$ pelas plantas de 156 linhagens de tomate foram consideradas também herdáveis sob a ação de genes aditivos (MAKMUR et alii, 1978).

A obtenção de técnicaș que facilitem a identificação e seleção de plantas mais eficientes na absorção e utilização de nutrientes, reveste-se de importância, em apoio a programas de melhoramento. 
O objetivo deste trabalho foi definir condições em solução nutritiva para diferenciação de genótipos de milho quanto à absorção e utilização de potássio e selecionar linhagens da coleção do Instituto Agronômico.

\section{MATERIAL E MÉTODOS}

\section{1 - Ensaio com seis linhagens de milho com niveis variados de $\mathrm{K}$ em solução nutritiva}

Este ensaio foi conduzido no Centro Experimental de Campinas, em agosto-setembro de 1983, em condições de casa de vegetação. As linhagens de milho utilizadas fazem parte da coleção do Instituto Agronômico. Aquelas de código inicial 10, 11 e 124 apresentam porte baixo e ciclo intermediário e as demais, porte alto e ciclo longo.

O sistema de crescimento de plantas utilizado foi o mesmo descrito em FURLANI et alii (1985a, b).

As sementes foram germinadas em rolos de papel de germinação e as plântulas, transplantadas aos dez dias de idade para vasos de 2,8 litros com solução nutritiva.

A composiçāo da solução nutritiva consistiu, basicamente, em miligrama/ litro: Ca: 151; Mg: 17; $\mathrm{N}-\mathrm{NO}_{3}: 125 ; \mathrm{N}-\mathrm{NH}_{4}: 20 ; \mathrm{P}: 6,0 ; \mathrm{Fe}: 3,6 ; \mathrm{Mn}: 0,5 ; \mathrm{B}: 0,3 ; \mathrm{Zn}$ : 0,15; Cu: 0,04 e Mo: 0,08. O potássio foi adicionado nas doses de 20,40,60, 80 e $100 \mathrm{mg} /$ litro de soluçăo. O S e o $\mathrm{Cl}$ variaram em, respectivamente, $28,32,36,40$ e $44 \mathrm{mg} /$ litro e $7,15,25,34$ e $43 \mathrm{mg} /$ litro com os niveis de $\mathrm{K}$. Esses nutrientes foram colocados na forma dos seguintes sais: $\mathrm{Ca}\left(\mathrm{NO}_{3}\right)_{2} .4 \mathrm{H}_{2} \mathrm{O}, \mathrm{MgSO}_{4} .7 \mathrm{H}_{2} \mathrm{O}$, $\mathrm{NH}_{4} \mathrm{NO}_{3}, \quad \mathrm{KH}_{2} \mathrm{PO}_{4}, \mathrm{KCl}, \mathrm{K}_{2} \mathrm{SO}_{4}, \quad \mathrm{MnCl}_{2} .4 \mathrm{H}_{2} \mathrm{O}, \quad \mathrm{H}_{3} \mathrm{BO}_{3}, \quad \mathrm{ZnSO}_{4} .7 \mathrm{H}_{2} \mathrm{O}$, $\mathrm{CuSO}_{4} \cdot 5 \mathrm{H}_{2} \mathrm{O}$ e $\left(\mathrm{NH}_{4}\right)_{6} \cdot \mathrm{Mo}_{7} \cdot \mathrm{O}_{24} \cdot 4 \mathrm{H}_{2} \mathrm{O} . \mathrm{O}$ ferro foi adicionado na forma de $\mathrm{Fe}$ -EDTA, preparado com Na-EDTA e FeSO ${ }_{4} \cdot 7 \mathrm{H}_{2} \mathrm{O}$. A composição da solução nutritiva foi baseada na desenvolvida por FURLANI (1981) e CLARK (1982).

Antes do transplante das plantas para as soluções-tratamentos, uma aliquota foi retirada de cada vaso, para análise da concentração de $\mathrm{K}$ por espectrofotometria de absorção atômica.

$\mathrm{O} \mathrm{pH}$ inicial da solução foi ajustado para 5,0, e durante o decorrer do experimento variou com o crescimento das plantas, decrescendo até cerca de 3,5 e aumentando depois gradativamente até $7,5 \pm 0,5$. Foi reajustado entre 5,0 e 5,5 em dias alternados, quando esteve acima desses valores, visto que, no cultivo em solução nutritiva, não se observaram problemas de deficiências ou danos às plantas, quando os valores de $\mathrm{pH}$ na solução estiveram dentro da faixa $3,5-5,5$ (FURLANI et alii, 1985a, b). A solução nutritiva foi continuamente arejada e não renovada, e os volumes dos vasos, completados diariamente com água destilada. $O$ delineamento experimental consistiu em blocos casualizados com quatro repe- 
tiçōes, e duas plantas por vaso. As médias das temperaturas máxima e mínima, na casa de vegetação, durante $o$ crescimento das plantas, foram $28,7 \pm 5,3^{\circ} \mathrm{C}$ e $15,0 \pm 1,8^{\circ} \mathrm{C}$ respectivamente.

As plantas foram colhidas com 34 dias de idade, após 24 dias em solução nutritiva, lavadas em água destilada e separadas em folhas, caules mais bainhas e raizes. A seguir, as amostras foram secas em estufa a $70^{\circ} \mathrm{C}$, pesadas (peso de materia seca), moídas e digeridas com mistura digestora nitroperclórica. Nos extratos, foram determinadas as concentrações de $\mathrm{K}, \mathrm{Ca}$ e $\mathrm{Mg}$ por espectrofotometria de absorção atômica, de acordo com o procedimento descrito por BATAGLIA et alii (1983).

\section{2 - Seleção de 37 linhagens de milho, quanto à absorção e utilização de K em solução nutritiva, com $20 \mathrm{mg} /$ /itro de $\mathrm{K}$}

Neste ensaio, conduzido no mesmo local, em setembro-outubro de 1983, utilizou-se o sistema descrito no anterior, com a mesma composiçāo básica de solução nutritiva e apenas um nivel de potássio, $20 \mathrm{mg} /$ litro.

Durante o ensaio, que consistiu em blocos casualizados com quatro repetiçōes, totalizando 148 vasos com duas plantas por vaso, as médias das temperaturas máxima e mínima, na casa de vegetaçáo, foram, respectivamente, $27,3 \pm 5,1^{\circ} \mathrm{C}$ e $11,6 \pm 1,6^{\circ} \mathrm{C}$.

As plantas foram colhidas aos 25 dias de idade e permaneceram 17 dias em solução, sendo processadas da forma descrita no ensaio anterior, para as determinações dos pesos de matéria seca e dos teores de $\mathrm{K}, \mathrm{Ca}$ e $\mathrm{Mg}$.

A avaliação estatística dos resultados foi feita através da análise da variância. Uma vez obtida significância no teste $F$ para a comparação entre linhagens, nos diversos parâmetros, elas foram classificadas de acordo com a produção de matéria seca total. Para isto, foi calculado o intervalo de confiança da mé dia geral das linhagens, com $99,9 \%$ de probabilidade e 36 graus de liberdade: $\bar{x} \pm t(0,001 ; 36) \cdot s(\bar{x})$.

A distribuição das médias, avaliada pelo índice de curtose, esteve dentro dos limites aceitáveis para uma distribuição normal.

As médias foram então enquadradas abaixo, dentro e acima do intervalo de confiança, distinguindo, respectivamente, três grupos de linhagens: ineficientes, medianamente eficientes e eficientes.

\section{RESULTADOS E DISCUSSÃO}

No primeiro ensaio, os resultados de produção de matéria seca mostraram uma pequena resposta no crescimento das linhagens 11.22.2.3 e 124.3.15.1, em função dos niveis de $\mathrm{K}$ na solução. Para as demais, essa resposta não ficou 
evidente, provavelmente devido às baixas temperaturas noturnas ocorridas no período, o que pode ter limitado a absorção de $\mathrm{K}$ pelas raizes nos niveis mais altos: registraram-se quinze noites com temperaturas inferiores a $15^{\circ} \mathrm{C}$ e nenhuma superior a 17. Entretanto, variaçōes entre linhagens foram observadas, quanto ao peso de matéria seca, destacando-se a $10.40 .1(\mathrm{~L}) \mathrm{com}$ melhor desenvolvimento da parte aérea no nível de $20 \mathrm{mg} /$ litro de $\mathrm{K}$ na solução (Quadro 1).Nesse nível, registrou-se a maior variação entre as médias das linhagens (CV $=19,1 \%$ ), e os sintomas de deficiência de $\mathrm{K}$ observados nas plantas caracterizaram-se por manchas amareladas e alongadas, que evoluíram da ponta para a parte média das folhas, resultando em áreas secas ou mortas.

QUADRO 1. Matéria seca produzida por seis linhagens de milho cultivadas em soluçāo nutritiva até aos 34 dias de idade, com 20, 40,60, 80 e $100 \mathrm{mg}$ /litro de $\mathrm{K}$ (duas plantas/vaso, quatro repetiçōes)

\begin{tabular}{|c|c|c|c|c|c|c|}
\hline \multirow{2}{*}{ Níveis de $K$} & \multicolumn{6}{|c|}{ Linhagens (1) } \\
\hline & 11.22 .2 .3 & $10.40 .1(\mathrm{~L})$ & 10.19.1.1 & 124.3 .15 .1 & 11.30 .1 .1 & 820 \\
\hline \multirow[t]{2}{*}{ mg/litro } & & & & & & \\
\hline & \multicolumn{6}{|c|}{ Matéria seca da parte aérea } \\
\hline 20 & 2,86 & 3,56 & 2,41 & 2,53 & 2,77 & 2,07 \\
\hline 40 & 3,10 & 3,86 & 3,05 & 3,03 & 3,27 & 2,33 \\
\hline 60 & 3,39 & 3,29 & 2,64 & 3,06 & 2,78 & 2,21 \\
\hline 80 & 3,34 & 3,52 & 3,11 & 3,17 & 3,10 & 2,22 \\
\hline 100 & 3,66 & 3,17 & 2,67 & 2,91 & 3,20 & 2,08 \\
\hline
\end{tabular}

Matéria seca de rázes

\begin{tabular}{|c|c|c|c|c|c|c|}
\hline 20 & 0,90 & 0,88 & 0,71 & 0,78 & 0,96 & 0,41 \\
\hline 40 & 0,92 & 0,97 & 0,97 & 0,86 & 0,99 & 0,45 \\
\hline 60 & 1,05 & 0,70 & 0,83 & 0,88 & 0,90 & 0,45 \\
\hline 80 & 1,05 & 0,85 & 1,00 & 0,88 & 0,94 & 0,46 \\
\hline \multirow[t]{2}{*}{100} & 1,10 & 0,72 & 0,81 & 0,77 & 1,06 & 0,42 \\
\hline & & \multicolumn{3}{|c|}{ Matéria seca total } & . & \\
\hline 20 & 3,76 & 4,44 & 3,13 & 3,31 & 3,73 & 2,48 \\
\hline 40 & 4,02 & 4,84 & 4,02 & 3,89 & 4,25 & 2,78 \\
\hline 60 & 4,44 & 3,99 & 3,46 & 3,94 & 3,69 & 2,66 \\
\hline 80 & 4,37 & 4,38 & 4,11 & 4,05 & 4,04 & 2,68 \\
\hline 100 & 4,76 & 3,90 & 3,48 & 3,69 & 4,26 & 2,50 \\
\hline
\end{tabular}

(1) Teste F significativo $(0,01)$. 
Os teores de $\mathrm{K}$ nas plantas foram crescentes em função dos tratamentos e mostraram-se mais elevados na parte aérea do que nas raizes. As plantas que se desenvolveram mais apresentaram teores menores de $\mathrm{K}$ nas folhas, evidenciando um efeito de diluição. $O$ conteúdo total de $K$ nas plantas explica melhor o comportamento observado quanto à resposta aos niveis de $\mathrm{K}$ na solução: algumas linhagens conseguiram absorver mais potássio que outras nos niveis mais elevados; nos niveis mais baixos, praticamente não houve diferença no conteúdo de $\mathrm{K}$ entre as linhagens, embora tenha havido diferenciação nos pesos de matéria seca (Quadro 2).

QUADRO 2. Teores de $\mathrm{K}$ nas partes das plantas, conteúdo total de $\mathrm{K}$ de seis linhagens de milho cultivadas em solução nutritiva até aos 34 dias de idade, com níveis variados de $\mathrm{K}$ (duas plantas/vaso, quatro repetiçōes)

\begin{tabular}{lllllll}
\hline \multirow{2}{*}{ Niveis de $\mathrm{K}$} & \multicolumn{5}{c}{ Linhagens (1) } \\
\cline { 2 - 7 } & 11.22 .2 .3 & $10.40 .1(\mathrm{~L})$ & 10.19 .1 .1 & 124.3 .15 .1 & 11.30 .1 .1 & 820 \\
\hline
\end{tabular}

mg/litro

$\begin{array}{rll}20 & 1,36 & 1,25 \\ 40 & 2,44 & 1,80 \\ 60 & 2,86 & 2,69 \\ 80 & 3,25 & 3,01 \\ 100 & 3,14 & 2,97\end{array}$

20

40

60

80

100

\section{0}

40

60

80

100

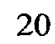

40

60

80

100

\section{1,33}

2,41

2,99

4,13

4,17

\section{0,99}

1,45

1,98

2,88

2,65

20

0

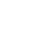

47,51
88,73
119,25
148,56
156,64

(1) Teste F significativo $(0,01)$
140,73

131,66
$K(\%)$ nas folhas

$\begin{array}{llll}1,66 & 1,62 & 1,25 & 1,73 \\ 2,35 & 2,37 & 2,00 & 2,56 \\ 3,01 & 2,79 & 2,88 & 2,99 \\ 3,54 & 3,31 & 3,35 & 3,08 \\ 3,27 & 3,33 & 3,68 & 3,53\end{array}$

$\mathrm{K}(\%)$ nos caules e bainhas

1,21
2,20
3,58
3,92
4,04

1,64

2,41

3,50

3,56

3,76

1,54

2,31

3,15

3,60

4,31

$K(\%)$ nas raizes

0,97

1,43

2,03

2,34

3,21

1,15

1,65

2,54

2,56

2,86

Conteúdo total de $\mathrm{K}$ (mg/duas plantas)

0,95

1,34

2,16

2,41

2,41
3,34

$\begin{array}{ll}1,52 & 1,99 \\ 2,42 & 3,04 \\ 3,48 & 3,81 \\ 4,25 & 4,01 \\ 4,23 & 4,40\end{array}$

52,56

48,06

47,65

88,35

82,58

105,47

108,26

135,99

129,57

115,80

132,90

0,93

1,31

2,28

2,42

2,92

1,48

1,99

2,99

2,87

3,07 . 
Os teores de $\mathrm{Ca}$ e $\mathrm{Mg}$ nas partes das plantas decresceram em função do aumento da concentração de $\mathrm{K}$ na solução nutritiva, evidenciando o já esperado efeito antagônico entre o $\mathrm{K}$ e esses cátions: quanto maior a concentração de $\mathrm{K}$ nas plantas, menores as de Ca e Mg (Quadro 3).

QUADRO 3. Teores de Ca e Mg nas partes das plantas de seis linhagens de milho cultivadas em soluçāo nutritiva até aos 34 dias de idade, com níveis variados de $\mathrm{K}$ (duas plantas/vaso, quatro repetiçōes)

\begin{tabular}{|c|c|c|c|c|c|c|c|c|c|c|c|c|}
\hline \multirow{3}{*}{ Níveis de $K$} & \multicolumn{12}{|c|}{ Linhagens $\left({ }^{1}\right)$} \\
\hline & \multicolumn{2}{|c|}{10.22 .2 .3} & \multicolumn{2}{|c|}{$10.40 .1(\mathrm{~L})$} & \multicolumn{2}{|c|}{10.19 .1 .1} & \multicolumn{2}{|c|}{124.3 .15 .1} & \multicolumn{2}{|c|}{11.30 .1 .1} & \multicolumn{2}{|c|}{820} \\
\hline & $\mathrm{Ca}$ & Mg & $\mathrm{Ca}$ & $\mathbf{M g}$ & $\mathrm{Ca}$ & $\mathbf{M g}$ & $\mathrm{Ca}$ & $\mathrm{Mg}$ & $\mathrm{Ca}$ & $\mathrm{Mg}$ & $\mathrm{Ca}$ & $\mathrm{Mg}$ \\
\hline mg/litro & \multicolumn{12}{|c|}{ Folhas } \\
\hline 20 & 0,84 & 0,54 & 0,90 & 0,68 & 0,71 & 0,40 & 0,90 & 0,69 & 1,04 & 0,93 & 0,99 & 0,74 \\
\hline 40 & 0,68 & 0,43 & 0,68 & 0,49 & 0,60 & 0,31 & 0,77 & 0,53 & 0,87 & 0,66 & 0,72 & 0,47 \\
\hline 60 & 0,60 & 0,33 & 0,66 & 0,37 & 0,53 & 0,26 & 0,68 & 0,41 & 0,74 & 0,44 & 0,81 & 0,51 \\
\hline 80 & 0,57 & 0,30 & 0,61 & 0,34 & 0,61 & 0,28 & 0,66 & 0,36 & 0,71 & 0,38 & 0,80 & 0,50 \\
\hline \multirow[t]{2}{*}{100} & 0,62 & 0,30 & 0,59 & 0,33 & 0,50 & 0,24 & 0,58 & 0,32 & 0,73 & 0,35 & 0,75 & 0,46 \\
\hline & \multicolumn{12}{|c|}{ Caules e bainhas } \\
\hline 20 & 0,87 & 0,89 & 0,75 & 0,72 & 0,72 & 0,58 & 0,84 & 0,95 & 0,93 & 1,15 & 0,86 & 1,00 \\
\hline 40 & 0,85 & 0,90 & 0,57 & 0,63 & 0,61 & 0,50 & 0,80 & 0,84 & 0,90 & 0,97 & 0,70 & 0,78 \\
\hline 60 & 0,69 & 0,65 & 0,55 & 0,45 & 0,45 & 0,35 & 0,62 & 0,59 & 0,73 & 0,62 & 0,57 & 0,59 \\
\hline 80 & 0,63 & 0,59 & 0,47 & 0,33 & 0,49 & 0,38 & 0,63 & 0,54 & 0,62 & 0,48 & 0,53 & 0,55 \\
\hline \multirow[t]{2}{*}{100} & 0,53 & 0,52 & 0,34 & 0,23 & 0,34 & 0,27 & 0,46 & 0,39 & 0,61 & 0,40 & 0,50 & 0,51 \\
\hline & \multicolumn{12}{|c|}{ Rá́zes } \\
\hline 20 & 0,48 & 0,65 & 0,50 & 0,75 & 0,39 & 0,49 & 0,54 & 0,92 & 0,58 & 1,05 & 0,41 & 0,80 \\
\hline 40 & 0,61 & 0,88 & 0,58 & 0,89 & 0,40 & 0,58 & 0,63 & 1,28 & 0,64 & 1,31 & 0,42 & 0,88 \\
\hline 60 & 0,39 & 0,56 & 0,44 & 0,88 & 0,25 & 0,20 & 0,50 & 1,03 & 0,51 & 0,99 & 0,37 & 0,52 \\
\hline 80 & 0,36 & 0,45 & 0,43 & 0,65 & 0,34 & 0,38 & 0,47 & 0,97 & 0,50 & 1,02 & 0,32 & 0,35 \\
\hline 100 & 0,36 & 0,32 & 0,30 & 0,21 & 0,26 & 0,13 & 0,43 & 0,63 & 0,40 & 0,55 & 0,31 & 0,28 \\
\hline
\end{tabular}

(1) Teste F significativo $(0,01)$.

$\mathrm{O}$ objetivo deste ensaio foi definir um nível relativamente baixo de $\mathrm{K}$, adequado para a diferenciação entre as linhagens, quanto à absorção e utilização deste nutriente, e, em vista dos resultados obtidos, optou-se pelo menor nivel, ou seja, $20 \mathrm{mg}$ /litro de $\mathrm{K}$, para o ensaio de seleção de linhagens.

Nos quadros 4,5 e 6 encontram-se os dados referentes ao segundo ensaio, quando se procedeu à seleção de 37 linhagens de milho, em solução nutritiva com $20 \mathrm{mg} / \mathrm{litro}$ de $\mathrm{K}$ colocados em ordem crescente de produção de matéria seca total. 
QUADRO 4. Matéria seca produzida por 37 linhagens de milho cultivadas em solução nutritiva até aos 25 dias de idade, com $20 \mathrm{mg} / \mathrm{litro}$ de $\mathrm{K}$ (duas plantas/vaso, quatro repetiçōes)

\begin{tabular}{|c|c|c|c|c|c|}
\hline \multicolumn{2}{|c|}{ Linhagens } & \multicolumn{3}{|c|}{ Matéria seca $\left({ }^{1}\right)$} & \multirow{2}{*}{$\begin{array}{c}\text { Classi- } \\
\text { ficação }{ }^{(2)}\end{array}$} \\
\hline № & Codigo & $\begin{array}{l}\text { Parte } \\
\text { aérea }\end{array}$ & Rafzes & Total & \\
\hline & & \multicolumn{3}{|c|}{ ـ g/duas plantas $\longrightarrow$} & \\
\hline 1 & Porto Rico 70.D.2 & 1,25 & 0,29 & 1,54 & I \\
\hline 4 & Ip 48.5.3 & 1,34 & 0,31 & 1,65 & I \\
\hline 30 & 10.13 .1 .1 & 1,73 & 0,35 & 2,08 & I \\
\hline 7 & Ip 321 & 1,90 & 0,42 & 2,31 & I \\
\hline 11 & $\operatorname{Pm} 624.2 .1$ & 2,00 & 0,47 & 2,47 & I \\
\hline 26 & 745 & 2,06 & 0,45 & 2,51 & I \\
\hline 23 & 532 & 2,07 & 0,48 & 2,55 & I \\
\hline 24 & 535.2 & 2,05 & 0,59 & 2,64 & I \\
\hline 36 & 11.19 .3 .3 & 2,27 & 0,48 & 2,75 & I \\
\hline 5 & Ip 365.4.1 & 2,26 & 0,50 & 2,76 & I \\
\hline 27 & 820 & 2,46 & 0,37 & 2,83 & I \\
\hline 13 & IA 2992.1.1.2.3 & 2,45 & 0,66 & 3,11 & ME \\
\hline 37 & 11.21 .1 .1 & 2,48 & 0,66 & 3,13 & ME \\
\hline 33 & 10.36 .1 & 2,67 & 0,56 & 3,22 & ME \\
\hline 2 & SLP 103.3.2 & 2,62 & 0,66 & 3,28 & ME \\
\hline 10 & Ip 421.2.1.1 & 2,74 & 0,73 & 3,47 & ME \\
\hline 35 & 11.01 .3 .1 & 3,11 & 0,56 & 3,66 & $\mathrm{ME}$ \\
\hline 18 & 490 & 2,92 & 0,76 & 3,68 & ME \\
\hline 20 & 519 & 3,10 & 0,70 & 3,80 & ME \\
\hline 6 & Ip 837.1.1.1 & 3,09 & 0,71 & 3,80 & $\mathrm{ME}$ \\
\hline 22 & 531 & 3,11 & 0,70 & 3,81 & $\mathrm{ME}$ \\
\hline 34-A & $10.40 .1(\mathrm{~A})$ & 3,08 & 0,72 & 3,81 & ME \\
\hline 41 & 11.24.1.1 & 2,95 & 0,89 & 3,83 & ME \\
\hline 12 & IA 2876.3 .1 .2 .3 & 3,26 & 0,69 & 3,94 & ME \\
\hline 44 & 723.4 & 3,20 & 0,86 & 4,06 & ME \\
\hline 28 & 10.8.2.1 & 3,07 & 1,02 & 4,09 & ME \\
\hline 43 & 124.3 .15 .1 & 3,50 & 0,92 & 4,42 & E \\
\hline 3 & Ip 701.1.1 & 3,40 & 1,04 & 4,44 & $\mathbf{E}$ \\
\hline 31 & 10.19 .1 .1 & 3,46 & 1,05 & 4,50 & $\mathrm{E}$ \\
\hline 16 & V 2017 Cuban Dent. & 3,86 & 0,78 & 4,64 & E \\
\hline 38 & 11.21 .5 .1 & 3,80 & 1,14 & 4,94 & $E$ \\
\hline 32 & 10.20 .3 .1 & 3,80 & 1,24 & 5,04 & $\mathrm{E}$ \\
\hline 42 & 11.30.1.1 & 4,11 & 1,05 & 5,16 & $\mathrm{E}$ \\
\hline 25 & 641.2 & 3,90 & 1,36 & 5,26 & $E$ \\
\hline 39 & 11.22.2.3 & 4,13 & 1,14 & 5,27 & $\mathrm{E}$ \\
\hline 29 & 10.11 .1 .1 & 4,14 & 1,20 & 5,33 & E \\
\hline $34-\mathrm{L}$ & $10.40 .1(\mathrm{~L})$ & 4,80 & 1,22 & 6,02 & $\mathbf{E}$ \\
\hline \multirow{3}{*}{\multicolumn{2}{|c|}{$\begin{array}{l}\text { CV \% (das médias) } \\
\text { Amplitude de variação } \\
\text { Intervalo de confiança (3) }\end{array}$}} & 28,5 & 38,9 & 30,1 & \\
\hline & & $1,25-4,80$ & $0,29-1,36$ & $1,54-6,02$ & \\
\hline & & & & $3,67 \pm 0,65$ & \\
\hline
\end{tabular}

(1) Teste $F$ significativo $(0,01)$ para diferenças entre linhagens, quanto à matéria seca da parte aérea, das rázes e total.

(2) $\mathrm{I}=$ ineficiente; $\mathrm{ME}=$ medianamente eficiente; $\mathrm{E}=$ eficiente.

(3) Intervalo de confiança para a media, calculado com $99,9 \%$ de probabilidade, numa distribuição $t$ $\bar{x} \pm t(0,001 ; 36 . s(\bar{x})$. 
QUADRO 5. Teores de $K$ nas partes das plantas, conteúdo de $K$ na planta inteira, relação parte aérea/raízes do conteúdo de $\mathrm{K}$, e relação de eficiência (matéria seca produzida/unidade de $\mathrm{K}$ absorvida) de 37 linhagens de milho cultivadas em solução nutritiva até aos 25 dias de idade, com $20 \mathrm{mg} /$ litro de $\mathrm{K}$ (duas plantas/vaso, quatro repetiçöes)

\begin{tabular}{|c|c|c|c|c|c|c|c|}
\hline \multicolumn{2}{|c|}{ Linhagens } & \multicolumn{3}{|c|}{ Teores de $K\left({ }^{1}\right)$} & \multicolumn{2}{|c|}{ Conteído de $K\left({ }^{1}\right)$} & \multirow{2}{*}{$\begin{array}{l}\text { Relação } \\
\text { de efi- } \\
\text { ciência }(1)\end{array}$} \\
\hline № & Código & Folhas & $\begin{array}{c}\text { Caules + } \\
\text { bainhas }\end{array}$ & Raízes & Total & $\begin{array}{c}\text { Parte área/ } \\
\text { /raízes }\end{array}$ & \\
\hline & & \multicolumn{3}{|c|}{$\%$} & $\mathbf{m g}$ & & mg MS/mg K \\
\hline 1 & Porto Rico 70.D.2 & 2,41 & 2,67 & 1,48 & 35,5 & 7,3 & 43,4 \\
\hline 4 & Ip 48.5 .3 & 2,52 & 2,95 & 1,83 & 40,2 & 6,3 & 40,8 \\
\hline 30 & 10.13 .1 .1 & 2,16 & 2,18 & 1,28 & 41,2 & 8,3 & 50,1 \\
\hline 7 & Ip 321 & 1,75 & 2,04 & 1,26 & 39,3 & 7,0 & 57,7 \\
\hline 11 & Pm 624.2.1 & 1,82 & 1,86 & 1,30 & 42,4 & 6,0 & 58,1 \\
\hline 26 & 745 & 1,90 & 1,92 & 1,44 & 44,0 & 5,8 & 56,9 \\
\hline 23 & 532 & 1,80 & 1,80 & 0,93 & 41,3 & 8,4 & 62,0 \\
\hline 24 & 535.2 & 1,94 & 1,55 & 0,98 & 41,3 & 6,8 & 63,1 \\
\hline 36 & 11.19 .3 .3 & 1,83 & 2,06 & 0,88 & 46,2 & 9,7 & 58,9 \\
\hline 5 & Ip 365.4.1 & 1,68 & 1,75 & 1,02 & 43,4 & 7,5 & 63,4 \\
\hline 27 & 820 & 1,59 & 1,83 & 1,02 & 45,1 & 10,9 & 62,6 \\
\hline 13 & IA 2992.1.1.2.3 & 1,55 & 1,77 & 0,94 & 45,6 & 6,5 & 68,4 \\
\hline 37 & 11.21.1.1 & 1,70 & 1,55 & 1,14 & 47,8 & 5,5 & 65,3 \\
\hline 33 & 10.36 .1 & 1,58 & 1,45 & 1,19 & 47,2 & 6,1 & 68,6 \\
\hline 2 & SLP 103.3.2 & 1,45 & 1,71 & 1,02 & 46,6 & 6,0 & 69,7 \\
\hline 10 & Ip 421.2.1.1 & 1,33 & 1,85 & 1,25 & 49,3 & 4,5 & 70,2 \\
\hline 35 & $11,01.3 .1$ & 1,50 & 1,40 & 0,74 & 46,4 & 10,2 & 77,5 \\
\hline 18 & 490 & 1,40 & 1,60 & 0,78 & 48,8 & 7,1 & 75,5 \\
\hline 20 & 519 & 1,36 & 1,37 & 0,84 & 47,9 & 7,2 & 79,2 \\
\hline 6 & Ip 837.1.1.1 & 1,23 & 1,60 & 0,85 & 48,2 & 7,0 & 79,1 \\
\hline 22 & 531 & 1,37 & 1,52 & 0,97 & 50,0 & 6,2 & 76,0 \\
\hline 34-A & $10.40 .1(\mathrm{~A})$ & 1,29 & 1,34 & 1,04 & 47,3 & 4,1 & 80,4 \\
\hline 41 & 11.24.1.1 & 1,45 & 1,27 & 0,92 & 47,2 & 5,0 & 80,4 \\
\hline 12 & IA 2876.3.1.2.3 & 1,42 & 1,38 & 0,90 & 51,8 & 7,5 & 76,3 \\
\hline 44 & 723.4 & 1,19 & 1,36 & 0,91 & 47,5 & 5,0 & 85,0 \\
\hline 28 & 10.8 .2 .1 & 1,22 & 1,29 & 0,95 & 47,6 & 3,9 & 86,0 \\
\hline 43 & 124.3 .15 .1 & 1,18 & 1,25 & 0,85 & 49,6 & 5,4 & 89,3 \\
\hline 3 & Ip 701.1.1 & 1,16 & 1,35 & 0,98 & 51,5 & 4,1 & 86,2 \\
\hline 31 & 10.19 .1 .1 & 1,19 & 1,13 & 0,89 & 49,4 & 4,4 & 90,7 \\
\hline 16 & V 2017 Cuban Dent. & 1,12 & 1,31 & 0,79 & 50,7 & 7,5 & 91,0 \\
\hline 38 & 11.21.5.1 & 1,01 & 1,19 & 0,88 & 50,7 & 4,2 & 97,4 \\
\hline 32 & 10.20 .3 .1 & 1,04 & 1,08 & 0,86 & 50,9 & 3,8 & 99,2 \\
\hline 42 & 11.30.1.1 & 1,01 & 1,25 & 0,69 & 52,0 & 6,1 & 99,4 \\
\hline 25 & 641.2 & 1,01 & 1,13 & 0,78 & 50,9 & 3,9 & 103,0 \\
\hline 39 & 11.22 .2 .3 & 1,01 & 1,14 & 0,80 & 52,2 & 4,7 & 100,7 \\
\hline 29 & $10,11,1,1$ & 1,07 & 1,03 & 0,75 & 52,6 & 4,9 & 101,6 \\
\hline 34-L & $10.40 .1(\mathrm{~L})$ & 0,86 & 1,03 & 0,80 & 53,7 & 5,8 & 112,3 \\
\hline \multirow{3}{*}{\multicolumn{2}{|c|}{$\begin{array}{l}\text { CV \% (das médias) } \\
\text { Coeficientes de correlaçāo (²) } \\
\text { Amplitude de variaçāo }\end{array}$}} & 34,6 & 27,5 & 28,6 & 9,0 & 33,8 & 23,0 \\
\hline & & $-0,94$ & $-0,88$ & $-0,66$ & 0,92 & $-0,51$ & 0,99 \\
\hline & & $0,86-2,52$ & $1,03-2,95$ & $0,80-1,83$ & $35,5-53,7$ & $3,8-10,9$ & $40,8-112,3$ \\
\hline
\end{tabular}

(1) Teste $\mathrm{F}$ significativo $(0,01)$ para diferenças entre linhagens quanto aos teores de $\mathrm{K}$ nas partes das plantas, quanto ao conteúdo total de $\mathrm{K}$ e à relação de eficiência.

(2) Coeficientes de correlação (r) entre os pesos de matéria seca das partes das plantas e os respectivos teores de $\mathrm{K}$; entre os pesos de matéria seca total e os conteúdos de $\mathrm{K}$ e relaçóes de eficiência. 
QUADRO 6. Teores de cálcio e magnésio nas partes das plantas de 37 linhagens de milho cultivadas em soluçäo nutritiva at aos 25 dias de idade, com $20 \mathrm{mg} /$ litro de K (duas plantas/vaso, quatro repetiçōes)

\begin{tabular}{|c|c|c|c|c|c|c|c|}
\hline \multicolumn{2}{|c|}{ Linhagens } & \multicolumn{3}{|c|}{ Teores de $\mathrm{Ca}\left({ }^{1}\right)$} & \multicolumn{3}{|c|}{ Teores de $\mathrm{Mg}\left({ }^{1}\right)$} \\
\hline \multirow[t]{2}{*}{ № } & Código & Folhas & $C+B\left({ }^{2}\right)$ & Raízes & Folhas & $C+B\left(^{2}\right)$ & Raízes \\
\hline & & \multicolumn{3}{|c|}{$\%$} & \multicolumn{3}{|c|}{$\longrightarrow \%-}$. \\
\hline 1 & Porto Rico 70.D.2 & 0,98 & 1,14 & 0,59 & 0,55 & 0,91 & 0,51 \\
\hline 4 & Ip 48.5 .3 & 0,45 & 0,65 & 0,63 & 0,37 & 0,93 & 0,77 \\
\hline 30 & 10.13 .1 .1 & 0,59 & 0,67 & 0,55 & 0,50 & 1,01 & 0,86 \\
\hline 7 & Ip 321 & 0,68 & 1,02 & 0,63 & 0,44 & 0,90 & 0,94 \\
\hline 11 & $\operatorname{Pm} 624.2 .1$ & 0,84 & 1,05 & 0,50 & 0,60 & 1,14 & 1,09 \\
\hline 26 & 745 & 0,96 & 1,14 & 0,72 & 0,80 & 1,27 & 0,73 \\
\hline 23 & 532 & 0,70 & 0,71 & 0,54 & 0,56 & 0,84 & 0,62 \\
\hline 24 & 535.2 & 0,94 & 0,78 & 0,71 & 0,82 & 1,06 & 0,72 \\
\hline 36 & 11.19 .3 .3 & 0,89 & 0,97 & 0,52 & 0,73 & 1,27 & 0,96 \\
\hline 5 & Ip 365.4.1 & 0,88 & 1,02 & 0,77 & 0,63 & 1,24 & 1,02 \\
\hline 27 & 820 & 0,85 & 0,82 & 0,49 & 0,84 & 1,10 & 0,74 \\
\hline 13 & IA 2992.1.1.2.3 & 0,65 & 0,87 & 0,60 & 0,60 & 1,11 & 0,84 \\
\hline 37 & 11.21.1.1 & 0,51 & 0,50 & 0,36 & 0,49 & 0,71 & 0,35 \\
\hline 33 & 10.36 .1 & 0,72 & 0,75 & 0,58 & 0,60 & 0,96 & 0,86 \\
\hline 2 & SLP 103.3.2 & 0,74 & 0,81 & 0,76 & 0,94 & 1,27 & 0,88 \\
\hline 10 & Ip 421.2.1.1 & 0,83 & 0,95 & 0,95 & 1,01 & 1,46 & 0,94 \\
\hline 35 & 11.01 .3 .1 & 0,64 & 0,84 & 0,80 & 0,60 & 1,04 & 0,86 \\
\hline 18 & 490 & 0,87 & 1,00 & 0,81 & 0,93 & 1,33 & 1,04 \\
\hline 20 & 519 & 1,03 & 1,20 & 0,68 & 0,70 & 1,07 & 0,81 \\
\hline 6 & Ip 837.1.1.1 & 1,14 & 1,15 & 0,69 & 1,02 & 1,37 & 0,97 \\
\hline 22 & 531 & 0,80 & 1,06 & 0,68 & 0,71 & 1,37 & 0,95 \\
\hline $34-A$ & $10.40 .1(\mathrm{~A})$ & 0,81 & 0,80 & 0,57 & 0,68 & 0,84 & 0,82 \\
\hline 41 & 11.24.1.1 & 0,93 & 0,98 & 0,60 & 0,73 & 1,18 & 1,16 \\
\hline 12 & IA 2876.3 .1 .2 .3 & 1,09 & 1,11 & 0,78 & 0,91 & 1,54 & 0,98 \\
\hline 44 & 723.4 & 0,88 & 0,99 & 0,83 & 1,00 & 1,28 & 1,01 \\
\hline 28 & 10.8 .2 .1 & 0,57 & 0,62 & 0,49 & 0,60 & 0,79 & 0,64 \\
\hline 43 & 124.3.15.1 & 0,89 & 0,96 & 0,72 & 0,82 & 1,12 & 0,96 \\
\hline 3 & Ip 701.1.1 & 0,90 & 0,92 & 0,72 & 0,96 & 1,37 & 1,00 \\
\hline 31 & 10.19 .1 .1 & 0,66 & 0,79 & 0,48 & 0,52 & 0,81 & 0,67 \\
\hline 16 & V 2017 Cuban Dent. & 0,99 & 0,90 & 0,58 & 1,33 & 1,65 & 0,74 \\
\hline 38 & 11.21.5.1 & 0,78 & 0,72 & 0,69 & 0,78 & 0,98 & 0,91 \\
\hline 32 & 10.20 .3 .1 & 0,94 & 0,87 & 0,68 & 0,92 & 1,14 & 0,88 \\
\hline 42 & 11.30 .1 .1 & 1,01 & 1,01 & 0,68 & 1,21 & 1,35 & 0,90 \\
\hline 25 & 641.2 & 1,01 & 1,06 & 0,85 & 1,03 & 1,34 & 0,79 \\
\hline 39 & 11.22 .2 .3 & 0,79 & 0,87 & 0,69 & 0,79 & 1,21 & 0,86 \\
\hline 29 & 10.11.1.1 & 0,86 & 0,74 & 0,82 & 0,84 & 1,03 & 1,01 \\
\hline 34-L & $10.40 .1(\mathrm{~L})$ & 0,96 & 0,86 & 0,71 & 0,96 & 1,03 & 0,90 \\
\hline \multirow{3}{*}{\multicolumn{2}{|c|}{$\begin{array}{l}\text { Média geral } \\
\text { CV das médias (\%) } \\
\text { Coeficientes de correlaçäo }\left({ }^{3}\right)\end{array}$}} & 0,83 & 0,90 & 0,66 & 0,77 & 1,14 & 0,86 \\
\hline & & 19,4 & 18,4 & 18,9 & 28,1 & 19,5 & 18,9 \\
\hline & & $-0,36$ & 0,03 & $-0,24$ & $-0,64$ & $-0,15$ & 0,04 \\
\hline
\end{tabular}

(1) Teste $\mathrm{F}$ significativo para diferenças entre linhagens quanto aos teores de $\mathrm{Ca}$ e $\mathrm{Mg}$ nas folhas.

(2) $\mathrm{C}+\mathrm{B}=$ colmos + bainhas.

(3) Coeficientes de correlaçāo ( $\mathrm{r}$ ) entre os teores de $\mathrm{K}$ nas partes das plantas e os teores de Ca e Mg. 
Observaram-se variações entre linhagens nos pesos de matéria seca da parte aérea e das raizes (teste $F$ significativo para $\alpha=0,01$ ), da ordem de $28,5 \mathrm{e}$ $38,9 \%$ (CV das médias) respectivamente (Quadro 4). As linhagens foram classificadas com base na produção de matéria seca total, utilizando-se de um intervalo de confiança para a média geral.

De acordo com esse intervalo de confiança, que variou de 3,02 a 4,32g de matéria seca, onze linhagens foram classificadas como ineficientes, quinze como medianamente eficientes, e onze como eficientes (Quadro 4). siâo da colheita.

$\mathrm{Na}$ figura 1, pode-se observar o aspecto de algumas linhagens por oca-

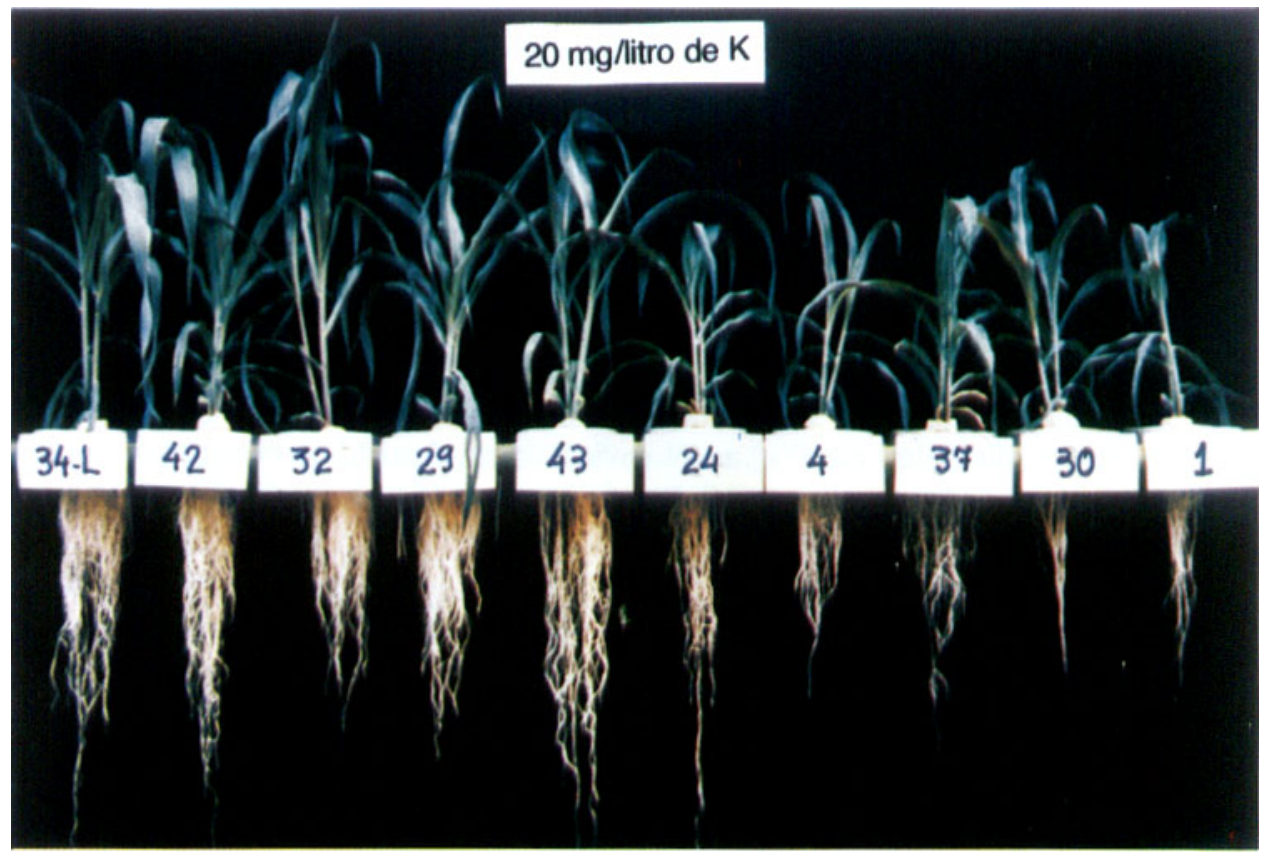

FIGURA 1. Aspecto de linhagens de milho cultivadas em solução nutritiva até aos 25 dias de idade, com $20 \mathrm{mg} /$ litro de $\mathrm{K}$

No quadro 5 , estão relacionados os teores de $\mathrm{K}$ nas partes das plantas, o conteúdo total de $\mathrm{K}$ e a relação de eficiência (matéria seca produzida por unidade de $\mathrm{K}$ absorvida) alinhados na mesma ordem dos dados de matéria seca total. Os teores de $\mathrm{K}^{\prime}$ decresceram com o maior desenvolvimento das plantas, evidenciando um acentuado efeito de diluição do nutriente nos tecidos. Houve também uma variação no conteúdo total de $\mathrm{K}$ nas plantas acompanhando a variação 
nos pesos de matéria seca total $(r=0,92)$, indicando diferenças entre as linhagens nas capacidades de absorçäo de $\mathrm{K}(\mathrm{CV}=9 \%)$. Maior variação ainda, porém, foi detectada para os valores de relação de eficiência ( $C V=23 \%)$, os quais se mostraram, também, altamente correlacionados com as variaçōes nos pesos de matéria seca $(r=0,99)$, indicando que o processo de utilizaçāo de $K$ nos tecidos das plantas foi tão ou mais limitante para o crescimento delas, do que o processo de absorção pelas raízes.

No quadro 6, encontram-se os teores de $\mathrm{Ca}$ e $\mathrm{Mg}$ nas partes das plantas: os de $\mathrm{Ca}$ mostraram certa variabilidade entre linhagens, que esteve pouco correlacionada com a variação observada nos teores de $K(r=-0,36$ para as folhas). Os teores de $\mathrm{Ca}$ foram mais elevados na parte aérea do que nas raízes. Entretanto, a variação observada nos teores de $\mathrm{Mg}$ nas folhas esteve mais correlacionada com a dos teores de $K(r=-0,64$, para as folhas), isto é, à medida que decresceram os teores de $\mathrm{K}$ nas linhagens mais desenvolvidas, aumentaram os de $\mathrm{Mg}$, indicando que ocorreu maior absorção de Mg por essas linhagens (Quadro 6).

As linhagens de código inicial 10,11 e 124 caracterizam-se, no campo, pelo porte baixo e ciclo intermediário, e as demais, pelo porte alto e ciclo longo. Verificou-se, entretanto, que essas características não tiveram influência no método de seleção utilizado neste trabalho, visto que as linhagens se distribuiram ao acaso ao longo da lista de classificação (Quadros 4, 5 e 6). $O$ mesmo foi observado na seleção dessas linhagens quanto à eficiência ao $\mathrm{P}$ e ao $\mathrm{N}$ (FURLANI et alii, 1985 a e b).

Nesses trabalhos, ficou evidenciado que linhagens eficientes ao $\mathrm{K}$ nem sempre foram eficientes ao $\mathrm{P}$ e ao $\mathrm{N}$ e vice-versa. Assim, os resultados obtidos com as linhagens parentais do híbrido duplo HD 7974 revelam o seguinte: a ip 48.5.3 mostrou-se ineficiente aos três nutrientes, N, P e K; a ip 365.4.1 mostrou-se ineficiente ao $\mathrm{N} \mathrm{e} \mathrm{K} \mathrm{e}$ intermediária ao $\mathrm{P}$; a lp 701.1.1, ineficiente ao $\mathrm{P}$, eficiente ao $\mathrm{K}$ e intermediária ao $\mathrm{N}$; e a lp 103.3.2, intermediária para os três nutrientes. Das linhagens classificadas como eficientes ao $\mathrm{K}$, salientaram-se as seguintes, que se mostraram eficientes também ao $\mathrm{P}$ e N: 10.40.1; 11.30.1.1; 641.2; 124.3.15.1; 11.22.2.3 e 10.11.1.1.

\section{CONCLUSŌES}

1. Foi possivel diferenciar os genótipos de milho quanto à absorção e utilização do potássio, com a técnica de crescimento de plantas em solução nutritiva. Definiu-se o nivel de $20 \mathrm{mg} / \mathrm{litro}$ de $\mathrm{K}$ para a seleção das linhagens, utilizando-se plantas com cerca de 30 dias de idade.

2. Das 37 linhagens utilizadas neste estudo, onze foram classificadas como ineficientes, quinze como medianamente eficientes e onze como eficientes na absorção e utilização do $\mathrm{K}$, em solução nutritiva com $20 \mathrm{mg} /$ /itro de $\mathrm{K}$. Não se observaram efeitos do porte e ciclo das linhagens sobre a classificação. 
3. Ambos os processos de absorção e utilização do $\mathrm{K}$ pelas plantas foram responsáveis pela variação observada entre as linhagens, mas o de utilização mostrou-se mais limitante que o de absorção no desenvolvimento das plantas.

\section{SUMMARY}

\section{DIFFERENTIAL GROWTH OF CORN INBRED LINES IN LOW POTASSIUM NUTRIENT SOLUTIONS}

Two experiments were conducted in the Experimental Station of Campinas, Instituto Agronomico, State of Sâo Paulo, Brazil, in 1983, under greenhouse conditions, in order to evaluate and select efficient corn (Zea mays L.) inbred lines in the uptake and use of potassium in nutrient solution. In a first trial, six lines were grow $\mathrm{n}$ with $20,40,60,80$ and $100 \mathrm{mg} \mathrm{K} / \mathrm{liter}$, up to thirty four days of age, w ith the objective of determining the adequate $\mathrm{K}$ level for plant grow th differentiation. In a second trial, thirty seven inbred lines were grown with $20 \mathrm{mg} \mathrm{K} / \mathrm{liter}$ up to twenty five days of age. Both experiments were arranged in randomized complete blocks with four replications, in 2.8 liter pots with two plants per pot. The nutrient solutions were continuously aerated, without renewing, and plants were allowed to grow till $\mathrm{K}$ deficiency symptoms appeared in the lower leaves. Variations among lines were observed for shoot and root dry weights, for total $K$ contents and dry matter produced per unit $\mathrm{K}$ absorbed (efficiency ratios). Larger variations among root systems (CV of means $=38.9 \%$ ) than shoots (CV of means $=$ 28.5\%) were observed. Genotypes were ranked and classified, according to the total dry matter yields, in efficient, intermediate and inefficient plants. Variations in total $\mathrm{K}$ contents $(\mathrm{CV}$ of means $=9.0 \%)$ and in efficiency ratios (CV of means $=23.0 \%$ ) showed high correlation with the total dry weights ( $r=0.92$ and 0.99 , respectively). Both $\mathrm{K}$ uptake and $\mathrm{K}$ use mechanisms seemed to be involved, but the larger variations among efficiency ratios indicated that the $\mathrm{K}$ utilization process $\mathrm{w}$ as the main factor limiting plant grow th.

Index terms: potassium, uptake, use, efficiency, corn inbred lines, nutrient solution.

\section{REFERÊNCIAS BIBLIOGRÁFICAS}

ANNUAL REPORT for 1980. Los Baños, Philippines, International Rice Research Institute, 1981. 467p.

BALIGAR, V.C. Potassium uptake by plants, as characterized by root density, species and K/Rb ratio. Plant \& Soil, The Hague, 85:43-53, 1985.

\& BARBER, S.A. Genotypic differences of corn for ion uptake. Agronomy Journal, Madison, 71:870-873, 1979.

BATAGLIA, O.C.; FURLANI, A.M.C.; TEIXEIRA, J.P.F.; FURLANI, P.R. \& GALLO, J.R. Análise química de plantas. Campinas, Instituto Agronômico, 1983. 31p. (Boletim técnico, 78) 
CLARK, R.B. Nutrient solution grow th of sorghum and corn in mineral nutrition studies. Journal of Plant Nutrition, New York, 5(8): 1039-1057, 1982.

- Plant genotype differences in the uptake, translocation, accumulation, and use of mineral elements required for plant growth. Plant \& Soil, The Hague, 72:175-196, 1983.

FRICK, H. \& BAUMAN, L.F. Heterosis in maize as measured by $\mathrm{K}$ uptake properties of seedling roots. Crop Science, Madison, 18:99-103, 1978.

_ \& - Heterosis in maize as measured by $\mathrm{K}$ uptake properties of seedling roots: pedigree analyses of inbreds with high or low augmentation potential. Crop Science, Madison, 19:707-710, 1979.

FURLANI, A.M.C. Differences in phosphorus uptake, distribution and use by sorghum genotypes grown with low phosphorus. Lincoln, University of Nebraska, 1981, $103 f$. Tese.(Doutoramento)

-; BATAGLIA, O.C. \& AZZINI, L.E. Comportamento diferencial de linhagens de arroz na absorção e utilização de nitrogênio em solução nutritiva. Revista Brasileira de Ciência do Solo, Campinas, 10(1):51-59, 1986.

; FURLANI, P.R.; AZZINI, L.E. \& CAMARGO, O.B.A. Avaliação de genótipos de arroz quanto à eficiência na utilização de fósforo em solução nutritiva e em solo. Revista Brasileira de Ciência do Solo, Campinas, 7:291-303, 1983.

—- \& LIMA, M. Eficiência de linhagens de milho na absorção e utilização de fósforo em solução nutritiva. Bragantia, Campinas, 44:129-147, 1985a.

-; —_ \& Diferenças entre linhagens de milho cultivadas em solução nutritiva quanto à absorção e utilização de nitrogênio. Bragantia, Campinas, 44(2):599-618, 1985 b.

-; CLARK, R.B.; MARANVILLE, J.W. \& ROSS, W.M. Root phosphatase activity of sorghum genotypes grow $n$ with organic and inorganic sources of phosphorus. Journal of Plant Nutrition, New York, 7(11):1583-1595, $1984 b$.

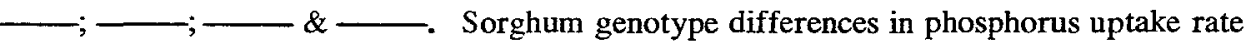
and distribution in plant parts. Journal of Plant Nutrition, New York, 7(7):1113-1126, 1984a.

GLASS, A.D.M. \& PERLEY, J.E. Varietal differences in potassium uptake by barley. Plant Physiology, Waltham, 65:160-164, 1980.

GLASS, A.D.M.; SIDDIQI, M.Y. \& GILES, K.I. Correlations between K uptake and hydrogen efflux in barley varieties. Plant Physiology, Waltham, 68:457-459, 1981.

MAKMUR, A.; GERLOFF, G.C. \& GABELMAN, W.H. Physiology and inheritance of efficiency in potassium utilization in tomatoes grown under potassium stress. Journal of American Society of Horticultural Science, Nex: York, 103(4):545-549, 1978.

OLIVEIRA, R.F. de \& MIELNICZUK, J. Caracterizaçāo de três cultivares de soja (Glycine max L. Merrill) quanto à eficiência de absorção e utilização de K. Agronomia Sulriograndense, Porto Alegre, 14(2):251-260, 1978.

SCHENK, M.K. \& BARBER, S.A. Potassium and phosphorus uptake by corn genotypes grown in the field as influenced by root characteristics. Plant \& Soil, The Hague, 54: $65-76,1980$.

SHEA, P.F.; GERLOFF, G.C. \& GABELMAN, W.H. Differing efficiencies of potassium utilization in strains of snapbeans, Phaseolus vulgaris L. Plant and Soil, The Hague, 28(2): 337-346, 1968. 